\title{
RETORNO AO ESPORTE APÓS ARTROPLASTIA TOTAL DO JOELHO: REVISÃO SISTEMÁTICA
}

RETURN TO SPORTS AFTER TOTAL KNEEARTHROPLASTY:A SYSTEMATIC REVIEW

RETORNO AL DEPORTEDESPUÉS DE LA ARTROPLASTIA TOTAL DE LA RODILLA: UNAREVISIÓN SISTEMÁTICA

Pedro Baches Jorge' (médico)

António de Pádua Silva Filho' (médico)

Rodrigo Yutaka Matsunaga' (médico)

Gabriel Abdo Elias Pecchia' (médico)

Jan Willem Cerf Sprey ${ }^{1}$ (médico) Luiz Gabriel Betoni Guglielmetti' (médico)

Marcos Vaz de Lima' (médico)

Ricardo de Paula Leite Cury ${ }^{1}$ (médico)

Aires Duarte Júnior' (médico)

1. Irmandade de Misericórdia da Santa Casa de São Paulo, Departamento de Ortopedia e Traumatologia, Grupo de Trauma do Esporte, São Paulo, SP, Brasil.

\section{Correspondência:}

Grupo de Trauma do Esporte Departamento de Ortopedia e Traumatologia Irmandade de Misericórdia da Santa Casa de São Paulo. Rua Cesário Mota Junior, 112, Vila Buarque, São Paulo, SP, Brasil. 01221-020.pbj1980@hotmail.com

\section{RESUMO}

Esta revisão sistemática da literatura teve como objetivo analisar as variáveis envolvidas no retorno às atividades esportivas em pacientes submetidos à artroplastia total do joelho (ATJ), verificar o tipo de esporte recomendado, o tempo de retorno ao esporte, a satisfação do paciente e o nível de atividade física após a cirurgia. Os estudos relacionados com a prática de atividade física e desportiva depois da ATJ foram selecionados por dois revisores independentes. Foram incluídos 11 estudos (10 transversais retrospectivos e um caso-controle). A maioria dos pacientes que participou de alguma atividade física regular antes da ATJ retornou à prática esportiva após a cirurgia, sendo mais comuns os esportes de baixo impacto como caminhada, esportes aquáticos, golfe, bicicleta estacionária, entre outros. Foi possível observar que o nível de atividade física não depende da dor e dos sintomas pós-operatórios, mas da satisfação com a cirurgia, motivação e capacidade funcional dos indivíduos. O tempo de retorno ao esporte variou de seis a 18 meses depois da ATJ. Apesar de alguns estudos mostrarem que os cirurgiões não recomendam a prática de esportes de alto impacto após a cirurgia, alguns estudos mostraram bons resultados, desde que o nível de atividade prévio à cirurgia, a população adequada e a expectativa do paciente no pós-operatório sejam considerados. Assim, conclui-se que o retorno ao esporte após ATJ não só é possível, como é recomendado em níveis recreativos, e um paciente colaborativo, instruído e com preparo adequado de condicionamento físico pode melhorar tanto no nível funcional e cardiovascular quanto no psicológico, com aumento da autoestima e da qualidade de vida. Há ainda a necessidade de estudos futuros, de boa qualidade metodológica como os ensaios clínicos randomizados, sobre os efeitos (benefícios e prejuízos) dos esportes de alto impacto para os pacientes submetidos à ATJ.

Descritores: artroplastia do joelho; exercício; esportes.

\section{ABSTRACT}

This systematic review of the literature aimed to analyze the variables involved in the return to sports activities in patients who underwent total knee arthroplasty (TKA), to verify the recommended type of sport, the time to return to sport, patient satisfaction and the level of physical activity after surgery. Studies related to the practice of physical and sports activity after TKA were selected by two independent reviewers. Eleven studies (10 retrospective cross-sectional and 1 case-control) were included. Most patients who participated in any regular physical activity before TKA returned to sports after surgery with low impact sports such as walking, water sports, golf, and stationary bicycle, among others. It was observed that the level of physical activity does not depend on pain and the postoperative symptoms, but on the satisfaction with the surgery, motivation, and functional capacity of individuals. The time take to return to sports ranged from six to 18 months after TKA. Although some studies show that surgeons do not recommend high-impact sports after surgery, some studies have shown good results, provided that the level of activity prior to surgery, the appropriate population and the patient's expectation in the postoperative period are all considered. Thus, it is concluded that the return to sports after TKA is not only possible, as it is recommended at recreational levels, and a collaborative, knowledgeable and well-trained patient can improve both the functional and cardiovascular level, as well as the psychological, with increased self-esteem and quality of life. There is also a need for further studies of good methodological quality as randomized clinical trials on the effects (benefits and damages) of high impact sports for patients who underwent TKA.

Keywords: arthroplasty, replacement, knee; exercise; sports.

\section{RESUMEN}

Esta revisión sistemática tuvo como objetivo analizar las variables que intervienen en el retorno a las actividades deportivas en los pacientes sometidos a artroplastia total de rodilla (ATR), comprobar el tipo recomendado de deporte, el tiempo para volver al deporte, la satisfacción del paciente y el nivel la actividad física después de la cirugía. Estudios relacionados con la práctica de la actividad física y deportiva después de la ATR fueron seleccionados por dos revisores independientes. Once estudios (10 transversales retrospectivos y 1 caso control) fueron incluidos. La mayoría de los pacientes que participaron en alguna actividad física regular antes de la ATR regresó al deporte después de la cirugía, siendo más comunes los deportes de bajo impacto, como la caminata, deportes acuáticos, golf, bicicleta estática, entre otros. Fue posible observar que el nivel de actividad física no depende del dolor ni de los síntomas postoperatorios, sino de la 
satisfacción con la cirugía, motivación y capacidad funcional de los individuos. El tiempo para volver al deporte varió de seis a 18 meses después de la ATR. Aunque algunos estudios muestran que los cirujanos no recomiendan la práctica de deportes de alto impacto después de la cirugía, algunos estudios mostraron buenos resultados, desde que sean considerados el nivel de actividad previo a la cirugía, la población adecuada y la expectativa del paciente en el postoperatorio. Por lo tanto, se concluye que el retorno al deporte después de la ATR no sólo es posible, como se recomienda en los niveles de recreo, y un paciente colaborador, bien informado y adecuadamente entrenado puede mejorar la aptitud tanto a nivel funcional y cardiovascular como psicológico, con aumento de la autoestima y calidad de vida. También existe la necesidad de más estudios de buena calidad metodológica como ensayos clínicos aleatorios sobre los efectos (beneficios y daños) de deportes de alto impacto para los pacientes sometidos a ATR.

Descriptores: artroplastia de reemplazo de rodilla; ejercicio; deportes.

\section{INTRODUÇÃO}

A artroplastia total do joelho (ATJ) é um procedimento de grande sucesso no tratamento das alterações degenerativas do joelho e apresenta uma demanda crescente em todo o mundo devido ao envelhecimento da população e à necessidade de preservar a qualidade de vida dos indivíduos. O objetivo da ATJ é o de restabelecer a forma e o alinhamento da articulação, devolvendo a estabilidade e a mobilidade, além de reduzir a dor e melhorar a função e qualidade de vida do paciente. Atualmente, mais de 450.000 artroplastias primárias de joelho são realizadas nos Estados Unidos ao ano, e estimativas apontam um aumento esperado de 673\% até o ano de 2030'.

Diante da ampla divulgação do sucesso clínico da substituição total da articulação dolorosa, e com a evolução da técnica cirúrgica e da qualidade do material de implante, houve um aumento considerável da demanda e das expectativas dos pacientes a respeito deste procedimento, tanto que muitos pacientes não só almejam a eliminação da dor, como também desejam praticar atividades físicas no pós-operatório, seja recreacional ou até mesmo competitiva ${ }^{1,2}$.

No entanto, não há ainda um consenso entre os cirurgiões em relação à permissão ou recomendação da prática esportiva após ATJ. Poucos estudos relatam que pacientes que retornaram ao esporte (de intensidade moderada a alta) após ATJ apresentaram maior desgaste da prótese, aumento do estresse na interface osso/implante e maior ocorrência de traumas, comparado aos que realizaram atividades físicas menos intensas 2,3 .

Lavernia et al. ${ }^{4}$ realizaram um estudo em cadáveres, observando que o desgaste volumétrico e linear do componente de polietileno da prótese está mais relacionado com o nível de atividade física praticada após a cirurgia e tempo de implante do que com a idade na qual o paciente realizou a cirurgia. Entretanto uma das limitações deste estudo é que os pacientes mais jovens eram portadores de doenças inflamatórias, como a artrite reumatoide e, portanto, praticavam menos atividade física.

De acordo com Jassim et al. ${ }^{5}$ os resultados dos estudos a respeito da participação de atividade esportivas após ATJ ainda é contraditório, sendo necessária a realização de estudos de melhor qualidade metodológica, que levem em consideração fatores como o tipo de esporte recomendado para as diferentes características dos pacientes e o tempo de seguimento de longo prazo, para que se possa determinar também os riscos de complicações e de desgaste do implante.

Sendo assim, o objetivo deste estudo foi analisar as variáveis envolvidas no retorno às atividades esportivas em pacientes submetidos à artroplastia total do joelho, verificar o tipo de esporte recomendado, o tempo de retorno ao esporte, a satisfação do paciente e o nível de atividade física após a cirurgia, além de encontrar dados referentes ao desgaste da prótese em praticantes de atividades físicas.

\section{METODOLOGIA}

Os estudos relacionados à prática de atividade física e desportiva após artroplastia total do joelho foram selecionados por dois revisores independentes, por meio da busca nas bases de dados eletrônicas: MEDLINE (Medical Literature Analysis and Retrieval System Online) via Pubmed, CENTRAL (Cochrane Central Register of Controlled Trials), via Wiley Cochrane Library e LILACS (Literatura Latino-Americana e do Caribe em Ciências da Saúde), via Biblioteca Virtual em Saúde (BVS). Foram excluídos os estudos que incluíram pacientes submetidos à artroplastia unicompartimental, os que analisaram artroplastia total de quadril e joelho associados, os estudos em cadáveres e as revisões narrativas ou sistemáticas. As estratégias de busca foram elaboradas utilizando os descritores de assunto: "Total Knee Arthroplasty", "Exercise", "Sports" e "Return to Sport", adaptados para cada base de dados (Quadro 1). Foi realizada também a busca manual pelas listas de referências dos estudos incluídos. A busca nas bases de dados foi realizada em julho de 2016. Não houve restrição de data ou idioma.

\section{RESULTADOS}

Foram encontrados 448 estudos por meio das buscas nas bases de dados: 397 na Medline (via Pubmed), sete na LILACS e 44 na CENTRAL. Após a retirada de 32 duplicações e análise de 416 títulos e resumos, 19 estudos apresentaram potencial de elegibilidade e foram lidos na íntegra. Desses, oito estudos foram excluídos (duas revisões narrativas, um estudo em cadáver, três estudos que incluíram apenas artroplastia unicompartimental e dois estudos que incluíram artroplastias de quadril e joelho), e 11 incluídos na revisão (Figura 1). O nível de evidência foi classificado de acordo com as recomendações do "Oxford Centre for Evidence-Based Medicine".

Dez dos onze estudos incluídos eram do tipo transversal retrospectivo (nível IV de evidência) e um do tipo caso-controle (nível III de evidência). O número total de pacientes incluídos nos estudos foi 3.013 submetidos à ATJ primária, sendo 1.558 do gênero masculino (51\%). A média de idade entre os participantes variou de 51 a 74 anos. O tempo de acompanhamento (follow up) para a avaliação dos desfechos variou em média entre dois e oito anos após a cirurgia. Na maioria dos estudos as avaliações

Quadro 1. Estratégias de busca para cada base de dados utilizada.

\begin{tabular}{c|c}
\hline Base de dados & Estratégia de busca \\
\hline MEDLINE & $\# 1$ (Arthroplasty, Replacement, Knee [Mesh]) \\
(via PUBMED) & $\begin{array}{c}\text { \#2 ((Sports [Mesh]) OR (Return to Sport } \\
{[\text { Mesh]) OR Exercise [Mesh])) }} \\
\text { LILACS }\end{array} \quad$ \#31 AND \#2 \\
(via BVS) & (mh:(Artroplastia do Joelho)) AND \\
\hline CENTRAL & (tw:(exercício) OR tw:(esportes)) \\
(via Cochrane & Replacement, Knee] explode all trees \\
Library) & \#2 MeSH descriptor: [Sports] explode all trees \\
& \#3 \#1 and \#2 \\
\hline
\end{tabular}


dos pacientes foram realizadas por telefone ou via email. Os principais desfechos analisados foram: o nível de atividade física (mensurado por escores validados como o University of California Los Angeles Activity Score (UCLA) e o Tegner Activity Score; a capacidade funcional do indivíduo, avaliada por escores como o Knee Society Score (KSS), o Oxford Knee Score (OKS), o Hospital for Special Surgery Score (HSS), o escore WOMAC e o Lysholm Knee Scoring Scale; a intensidade da dor, mensurada pela Escala Analógica Visual (VAS); e a satisfação do paciente. Questionários sobre as habilidades esportivas específicas também foram utilizados para avaliar o tipo e intensidade da atividade esportiva praticada. As características dos estudos incluídos estão detalhadas na Tabela $1^{7-17}$.

\section{DISCUSSÃO}

A ATJ é um dos procedimentos ortopédicos mais realizados em todo o mundo e tem como principais objetivos o alívio da dor e a melhora da função do paciente. No entanto, com o aumento da expectativa de vida e da qualidade de vida e de saúde das pessoas com idade avançada, é possível observar uma maior preocupação desses indivíduos com a prática regular de atividades físicas ou desportivas, com o intuito de melhorar a qualidade de vidas global. ${ }^{18}$ Os benefícios físicos e mentais da atividade física são bem estabelecidos na literatura, e os pacientes com osteoartrite são comumente prejudicados nesse aspecto devido às limitações funcionais impostas pela doença. Uma vez que a articulação acometida é substituída pela prótese esses indivíduos sentem-se encorajados a realizar tanto as atividades que praticavam antes da doença quanto novas modalidades. Sabe-se ainda que os exercícios físicos e desportivos praticados regularmente estão relacionados à redução da ansiedade e da depressão, e à melhora do perfil cardiovascular e da qualidade óssea do indivíduo. Entretanto, pouco se sabe ainda sobre o tipo e intensidade de atividade esportiva a ser recomendada para esses pacientes. ${ }^{718} \mathrm{O}$ objetivo do presente estudo foi analisar as variáveis envolvidas no retorno às atividades esportivas em pacientes submetidos à ATJ.

Na amostra analisada pelos onze estudos incluídos foi possível observar predominância do gênero masculino (51\%) em relação ao feminino. Fato que pode ser explicado pelos achados de estudos que mostraram maior número de homens praticantes de atividades esportivas após a ATJ, especialmente as de alto impacto como tênis, corrida, entre outras. ${ }^{10,11,15}$

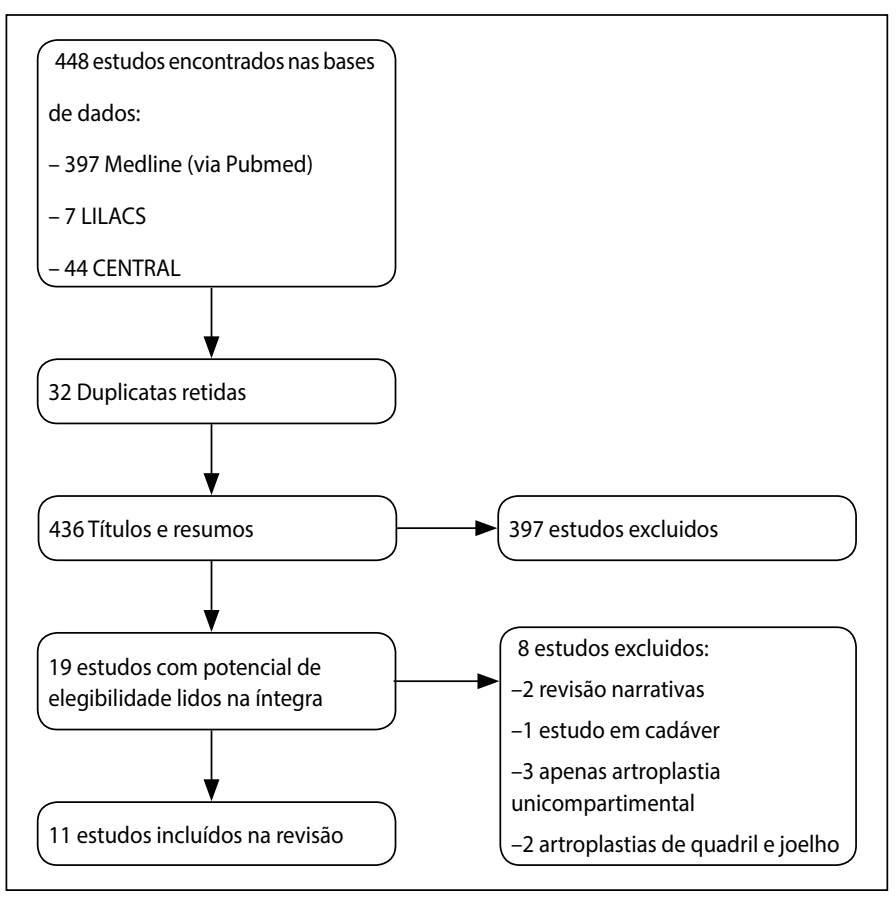

Figura 1. Fluxograma do processo de identificação e seleção dos estudos.
A média de idade variou entre 51 e 74 anos, e o estudo de Bonnin et al. ${ }^{8}$ demonstrou que entre os indivíduos com idade até 75 anos, 56\% praticavam um ou mais esportes considerados leves (bicicleta estacionária, alongamento, natação e golfe), 66\% praticavam um ou mais esportes intermediários (jardinagem - gardening, caminhadas, ginástica, musculação e dança), e apenas 10\% relataram participar regularmente um ou mais esportes vigorosos (esqui, ténis, squash e corrida de mais de 500 metros). Esses autores puderam ainda observar que a ausência nas atividades esportivas não foi relacionada especificamente aos sintomas do joelho como dor ou desconforto, e sim à motivação e satisfação do paciente, ou seja, no grupo de pacientes motivados a frequência de participação nos esportes foi maior. Embora apenas 10\% de todos os pacientes praticavam regularmente esportes vigorosos, esta taxa é de $63 \%$ no subgrupo de pacientes motivados.

O tempo de retorno ao esporte após a cirurgia foi descrito em apenas três estudos ${ }^{9,13,17}$, e variou de seis a 18 meses. Vale ressaltar que esses três estudos incluíram apenas pacientes praticantes de atividade esportiva antes da cirurgia (golfe e tênis). Nove dos onze estudos ${ }^{7-9,11,12,14-17}$ relataram que a maioria dos pacientes que retornaram à prática esportiva após ATJ participavam mais frequentemente de esportes de baixo impacto, como caminhada e natação, do que os de alto impacto. De acordo com Mont et al. ${ }^{10}$ é possível para alguns pacientes praticarem atividades esportivas extenuantes após ATJ. Esses autores relataram uma série de pacientes com média de 66 anos de idade, que praticavam algum esporte de alto impacto como o tênis, corrida, esqui, squash e basquete, por pelo menos quatro vezes por semana (média de 3,5 horas por semana). Ao final de quatro anos de follow up dos 33 joelhos operados, 32 apresentavam bom sinal radiográfico e clínico.

Outro estudo de Mont et al. ${ }^{13}$ avaliou jogadores de tênis em nível competitivo submetidos à ATJ quando tinham em média 57 anos. Após sete anos de seguimento todos voltaram a jogar no mesmo nível prévio à cirurgia (pelo menos três vezes por semana, com dor relatada em apenas 12\%). Todos estavam satisfeitos com o resultado da cirurgia em relação ao retorno ao esporte. Contudo, apesar desse estudo ter apresentado bons resultados, deve ser analisado com cautela, pois a população foi composta por participantes selecionados e os achados não se aplicam a todos os desportistas. A maioria dos cirurgiões ainda tem receio de recomendar o tênis em nível competitivo após ATJ. ${ }^{13,18}$

Bradbury et al. ${ }^{14}$ e Chang et al. ${ }^{7}$, relataram em seus estudos que os pacientes que praticavam atividade esportiva regular antes da cirurgia estavam mais propensos a retornar ao esporte e relataram maior satisfação com o resultado da cirurgia do que os pacientes menos ativos. Esta motivação pode ajudar também na reabilitação pós-operatória, e consequentemente facilitar o retorno ao esporte. Esses achados corroboram com o estudo de Healy et al. ${ }^{1}$, que além disso, descreve que a reabilitação e os programas de fortalecimento muscular para o tipo de esporte a qual o paciente pretende praticar ajudam a proteger a prótese e evitam traumas que poderiam desencadear possíveis fraturas periprotéticas, solturas precoces ou revisão prematura.

Muitos fatores podem contribuir para a taxa de sobrevivência da prótese, tais como as técnicas operatórias corretas, comorbidades associadas, grau de atividade física após a cirurgia e a espessura do componente de polietileno. Componentes com menos de 7,5 milímetros de espessura levam a maiores desgastes. Assim como a geometria relativa da superfície posterior das próteses ajudam a reduzir o estresse de contato, diminuindo assim o desgaste do polietileno, principalmente quando comparados às antigas configurações flat-on-flat.19 $\mathrm{A}$ cimentação também ajuda a redução de soltura asséptica por prover uma barreira aos debris de polietileno, assim como o alinhamento da prótese e ligamentar ajudam a prevenir uma carga excêntrica. ${ }^{20}$ 
Tabela 1. Características dos estudos incluídos.

\begin{tabular}{|c|c|c|c|c|}
\hline Estudo/ ano & $\begin{array}{l}\text { Tipo de estudo/ } \\
\text { follow up }\end{array}$ & $\begin{array}{l}\text { Amostra/ } \\
\text { gênero/ } \\
\text { média de idade }\end{array}$ & Desfechos avaliados & Resultados relevantes \\
\hline $\begin{array}{c}\text { Chang et al. } \\
2014\end{array}$ & $\begin{array}{l}\text { Transversal } \\
\text { retrospectivo } \\
\text { (nível IV) } \\
2 \text { anos }\end{array}$ & $\begin{array}{l}\mathrm{N}=369 \\
\text { (ATJ primária) } \\
339 \mathrm{~F} / 30 \mathrm{M} \\
68,8 \text { anos }\end{array}$ & $\begin{array}{l}\text { - Nível de atividade } \\
\text { física e desportiva } \\
\text { - Satisfação do } \\
\text { paciente } \\
\text { - Função } \\
\text { - Dor }\end{array}$ & $\begin{array}{c}\text { - Atividades mais comuns antes e após ATJ: caminhada } \\
(59,9 \%), \text { natação }(23 \%) \text { e ciclismo }(21,7 \%) \text {. } \\
\text { - UCLA: aumentou após ATJ } \\
\text { - Houve redução na participação de esportes de alto impacto } \\
\text { - Correlação entre satisfação do paciente e prática regular de atividade física } \\
\text { - Não relatou tempo de retorno ao esporte }\end{array}$ \\
\hline $\begin{array}{c}\text { Bonnin et al. }{ }^{8} \\
2010\end{array}$ & $\begin{array}{l}\text { Transversal } \\
\text { retrospectivo } \\
\text { multicêntrico } \\
\quad \text { (nível IV) } \\
\quad 3,6 \text { anos }\end{array}$ & $\begin{array}{l}\mathrm{N}=347 \\
\text { (ATJ primária) } \\
227 \mathrm{~F} / 120 \mathrm{M} \\
\text { 74,8 anos }\end{array}$ & $\begin{array}{l}\text { - Nível de atividade } \\
\text { física e desportiva } \\
\text { - Satisfação do } \\
\text { paciente } \\
\text { - Função }\end{array}$ & $\begin{array}{l}\text { - 66\% relataram estar tão ativos quanto antes da ATJ } \\
-83 \% \text { dos pacientes satisfeitos } \\
\text { - Correlação entre satisfação e motivação do paciente, função e participação esportiva } \\
-<75 \text { anos: 10\% participavam de esportes vigorosos após ATJ } \\
\text { - Não relatou tempo de retorno ao esporte }\end{array}$ \\
\hline $\begin{array}{l}\text { Jackson } \\
\text { et al. }^{9} \\
2009\end{array}$ & $\begin{array}{l}\text { Transversal } \\
\text { retrospectivo } \\
\text { (nível IV) } \\
\text { 8,7 anos }\end{array}$ & $\begin{array}{c}\mathrm{N}=93 \\
\text { (praticantes de golfe } \\
\text { amador - ATJ primária) } \\
19 \mathrm{~F} / 74 \mathrm{M} \\
66 \text { anos }\end{array}$ & $\begin{array}{l}\text { - Nível de atividade } \\
\text { física e desportiva } \\
\text { - Tempo de retorno } \\
\text { ao esporte }\end{array}$ & $\begin{array}{c}\text { - 57\% retornaram ao esporte } 6 \text { meses após ATJ } \\
\text { - 81\% relataram frequência da prática esportiva maior } \\
\text { ou igual ao período antes da cirurgia } \\
\text { - Menos dor durante o golfe comparado ao período antes da cirurgia }\end{array}$ \\
\hline $\begin{array}{c}\text { Mont et al. }{ }^{10} \\
2008\end{array}$ & $\begin{array}{l}\text { Transversal } \\
\text { retrospectivo } \\
\text { (nível IV) } \\
\text { 3,7 anos }\end{array}$ & $\begin{array}{c}\mathrm{N}=31 \\
\text { (praticantes de } \\
\text { esportes de alto } \\
\text { impacto - ATJ primária) } \\
\begin{array}{c}10 \mathrm{~F} / 21 \mathrm{M} \\
66 \text { anos }\end{array}\end{array}$ & $\begin{array}{l}\text { - Nível de atividade } \\
\text { física e desportiva } \\
\text { - Função } \\
\text { - ADM }\end{array}$ & $\begin{array}{l}\text { - Esportes mais praticados: tênis, corrida, aeróbica, esqui e squash } \\
\qquad \begin{array}{l}\text {-96\%: KSS bom ou excelente } \\
\text { - ADM média: } 119^{\circ}\end{array} \\
\text { - Não relatou tempo de retorno ao esporte }\end{array}$ \\
\hline $\begin{array}{c}\text { Dahm et al. }{ }^{11} \\
2008\end{array}$ & $\begin{array}{l}\text { Transversal } \\
\text { retrospectivo } \\
\text { (nível IV) } \\
\text { 5,7 anos }\end{array}$ & $\begin{array}{l}\mathrm{N}=1630 \\
\text { (ATJ primária) } \\
662 \mathrm{~F} / 544 \mathrm{M} \\
67 \text { anos }\end{array}$ & $\begin{array}{l}\text { - Nível de atividade } \\
\text { física e desportiva } \\
\text { - Função }\end{array}$ & $\begin{array}{l}- \text {-91\% satisfeitos com o nível de atividade } \\
- \text { KSS: bom ou excelente } \\
\text { - UCLA: participação regular em atividades moderadas } \\
\text { - Houve correlação dos melhores resultados de UCLA, KSS e o gênero masculino } \\
->70 \text { anos }<\text { UCLA } \\
-16 \% \text { esportes de alto impacto } \\
\text { - Não relatou tempo de retorno ao esporte }\end{array}$ \\
\hline $\begin{array}{l}\text { Chatterji } \\
\text { et al. }^{12} \\
2005\end{array}$ & $\begin{array}{l}\text { Transversal } \\
\text { retrospectivo } \\
\text { (nível IV) } \\
2 \text { anos }\end{array}$ & $\begin{array}{l}\mathrm{N}=144 \\
\text { (ATJ primária) } \\
64 \mathrm{~F} / 80 \mathrm{M} \\
70,8 \text { anos }\end{array}$ & $\begin{array}{l}\text { - Nível de atividade } \\
\text { física e desportiva } \\
\text { - Função }\end{array}$ & $\begin{array}{l}\text { - 70\% continuaram a prática de atividade física após ATJ } \\
\text { - Houve aumento na prática de caminhada e esportes aquáticos, } \\
\text { sendo os primeiros a serem praticados logo após ATJ } \\
\text { - Não relatou tempo de retorno ao esporte }\end{array}$ \\
\hline $\begin{array}{c}\text { Mont et al. }{ }^{13} \\
2002\end{array}$ & $\begin{array}{l}\text { Transversal } \\
\text { retrospectivo } \\
\text { multicêntrico } \\
\text { (nível IV) } \\
\quad 7 \text { anos }\end{array}$ & $\begin{array}{l}\text { N= } 33 \text { (praticantes } \\
\text { de tênis competitivo } \\
\text { - ATJ primária) } \\
5 \text { F / } 28 \text { M } \\
64 \text { anos }\end{array}$ & $\begin{array}{l}\text { - Capacidade } \\
\text { funcional } \\
\text { - Satisfação do } \\
\text { paciente }\end{array}$ & $\begin{array}{c}\text { - Todos retornaram ao esporte em média após 5,9 meses da ATJ; } 2 \text { a } 3 \text { vezes por semana } \\
\text { - Houve redução da dor pré e pós ATJ, durante o jogo de tênis } \\
\text { - Todos os participantes ficaram satisfeitos com a cirurgia }\end{array}$ \\
\hline $\begin{array}{l}\text { Bradbury } \\
\text { et al. }^{14} \\
1998\end{array}$ & $\begin{array}{l}\text { Transversal } \\
\text { retrospectivo } \\
\text { (nível IV) } \\
5 \text { anos }\end{array}$ & $\begin{array}{c}\mathrm{N}=160 \\
\text { (ATJ primária - } \\
208 \text { joelhos) } \\
88 \mathrm{~F} / 72 \mathrm{M} \\
73 \text { anos }\end{array}$ & $\begin{array}{l}\text { - Nível de atividade } \\
\text { física e desportiva }\end{array}$ & $\begin{array}{l}\text { - 65\% que praticavam esportes antes da cirurgia retornaram às atividades } \\
\text { - Esportes mais comuns: de baixo impacto (golfe) } \\
\text { - Os pacientes não foram encorajados a participar de esportes de alto impacto } \\
\text { - Não relatou tempo de retorno ao esporte }\end{array}$ \\
\hline $\begin{array}{l}\text { Diduch } \\
\text { et al. }^{15} \\
1997\end{array}$ & $\begin{array}{l}\text { Transversal } \\
\text { retrospectivo } \\
\text { (nível IV) } \\
8 \text { anos }\end{array}$ & $\begin{array}{c}\text { N }=80 \\
\text { (ATJ primária - } \\
103 \text { joelhos) } \\
\text { Gênero não descrito } \\
51 \text { anos }\end{array}$ & $\begin{array}{l}\text { - Nível de atividade } \\
\text { física e desportiva } \\
\text { - Função }\end{array}$ & $\begin{array}{c}\text { - Houve melhora da função: } \\
\text { KSS e HSS: bom ou excelente } \\
\text { - Houve melhora do nível de atividade física, avaliado pelos escores Tegner e Lysholm } \\
\text { - Atividades mais realizadas (>60\%): caminhadas diárias } \\
\text { (em média } 3 \text { km), bicicleta estacionária } \\
\text { - 23,7\% retornaram aos esportes de alto impacto (ciclismo competitivo, esqui e tênis) } \\
\text { - Não relatou tempo de retorno ao esporte }\end{array}$ \\
\hline $\begin{array}{c}\text { Ries et al. }{ }^{16} \\
1996\end{array}$ & $\begin{array}{l}\text { Caso-controle } \\
\text { (nível III) } \\
2 \text { anos }\end{array}$ & $\begin{array}{l}\text { Casos: } \mathrm{N}=16 \\
\text { ATJ primária } \\
\text { Controle: } \mathrm{N}=16 \\
\text { OA de joelho } \\
20 \mathrm{~F} / 12 \mathrm{M} \\
67 \text { anos }\end{array}$ & \begin{tabular}{|c|} 
- Aptidão \\
cardiovascular (teste \\
de esforço máximo: \\
bicicleta ergométrica) \\
- Nível de atividade \\
física e desportiva
\end{tabular} & $\begin{array}{l}\text { - Houve melhora significativa da função cardiopulmonar } \\
\text { e da atividade física, } 1 \text { e } 2 \text { anos após a ATJ } \\
\text { - Houve piora dos desfechos nos pacientes do grupo controle após } 1 \text { e } 2 \text { anos } \\
\text { - Não relatou tempo de retorno ao esporte }\end{array}$ \\
\hline $\begin{array}{l}\text { Mallon } \\
\text { et al. }^{17} \\
1993\end{array}$ & $\begin{array}{l}\text { Transversal } \\
\text { retrospectivo } \\
\text { (nível IV) } \\
\text { 4,7 anos }\end{array}$ & $\begin{array}{c}\mathrm{N}=83 \\
\text { (praticantes de golfe } \\
\text { amador - ATJ primária) } \\
21 \mathrm{~F} / 62 \mathrm{M} \\
65,4 \text { anos }\end{array}$ & $\begin{array}{l}\text { - Nível de atividade } \\
\text { física e desportiva } \\
\text { - Achados } \\
\text { radiográficos } \\
\text { - Dor }\end{array}$ & 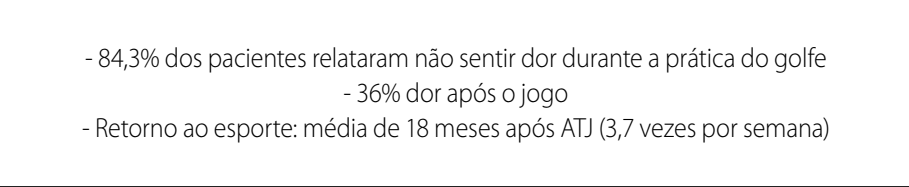 \\
\hline
\end{tabular}


Outra observação a ser feita é que segundo Lavernia et al. ${ }^{4} \mathrm{com}$ o decorrer do tempo quanto maior o tempo de implante menor o desgaste do componente de polietileno. Isso pode ser explicado por dois fatores: o primeiro é que com o avançar da idade o indivíduo torna-se mais sedentário, e a segunda é que com o tempo o desgaste do polietileno na prótese flat-on-flat vai tendo um ajuste melhor entre as superfícies e diminuindo o desgaste por ciclo.

No estudo de Mallon e Callaghan 17, o qual avaliou 83 jogadores de golfe amador com uma média de follow-up de 4,7 anos após ATJ, foi possível correlacionar um maior desgaste no joelho esquerdo em golfistas destros, devido à biomecânica do giro na tacada. Por ser um esporte de menor impacto, os cirurgiões estão mais sujeitos a liberarem o golfe como esporte a ser praticado após a ATJ, apesar desse esporte não ser uma realidade no Brasil. Além desse, outros estudos incluídos nessa revisão relataram que dentre os tipos de esportes mais praticados, os mais comuns foram os de baixo impacto como a caminhada, golfe, natação e ciclismo (bicicleta estacionária) ${ }^{5,21}$. No estudo de Chatterji et al. ${ }^{12}, 70 \%$ dos pacientes continuaram a prática de atividade física após a artroplastia, e houve um aumento na prática de caminhada e esportes aquáticos, sendo os primeiros esportes a serem praticados após o procedimento. Apesar do consenso geral de que se deve dar preferência para atividades de baixo impacto, ainda não há evidencias que corroboram com essa afirmativa. Com uma amostra expressiva de 1630 pacientes, Dahm et al. ${ }^{11}$, analisaram que 91\% dos pacientes estavam satisfeitos com o nível esportivo após a prótese, e somente $11 \%$ referiu praticar atividades esportivas mais extenuantes, 5 anos após a cirurgia.

Outro aspecto importante é que devido ao quadro álgico nos pacientes com osteoartrose de joelho, sua prática de atividade física também fica limitada, o que acarreta em queda do perfil cardiovascular dos pacientes. Em seu estudo caso-controle, Ries et al. ${ }^{16}$ demonstraram esse perfil cardiovascular ao comparar em um estudo caso-controle, os pacientes submetidos à ATJ com pacientes com diagnóstico de osteoartrite. Os pacientes com osteoartrite do joelho tratados clinicamente tiveram um declínio no seu perfil cardiovascular e na eficiência do sistema musculoesquelético, provavelmente decorrente das limitações proveniente da patologia, enquanto que os submetidos à cirurgia tiveram uma melhora significativa no seu perfil cardiovascular.

Portanto podemos presumir que o retorno ao esporte após ATJ não só é possível, mas como é recomendado em níveis recreativos e em alguns casos competitivo, e que um paciente colaborativo, instruído e com um preparo adequado de condicionamento físico pode melhorar tanto a parte psicológica com melhora da autoestima bem como sua saúde cardiovascular.

Apesar de haver melhora da condição cardiovascular, naqueles pacientes com ATJ que retornam à atividade física, não é descrito na literatura que níveis excelentes de condicionamento físico aeróbico conseguem ser atingidos após a cirurgia de plastia articular22.

Faltam dados na literatura que demonstrem com clareza a recuperação total de condicionamento físico após a ATJ, principalmente por ausência de descrição das imitações clínicas impostas pela presença da prótese ${ }^{22}$.

Nos estudos levantados nesta revisão a maioria dos autores recomendam esportes de baixo impacto e não recomendam os de alto impacto após ATJ. De acordo com a revisão de Seiler et al. ${ }^{18}$ os cirurgiões ortopedistas, por consenso, recomendam a participação em esportes de baixo impacto, como: natação, golfe, bicicleta, boliche, e mergulho; ao contrário dos esportes de alto impacto, tais como corrida, esqui aquático, hockey, handebol, caratê e futebol são desencorajados. Entretanto, na maioria das vezes essas recomendações foram baseadas em suas experiências pessoais ao invés de evidencias científicas de qualidade. Ainda neste contexto, vários estudos têm sido publicados e relatam bons resultados funcionais a respeito desse nível de atividade de alto impacto, em populações específicas ${ }^{18}$. Portanto, para evitar expectativas irreais, é importante analisar com precisão os desejos de cada paciente e seus hábitos desportivos reais antes e após a ATJ, para que as recomendações adequadas possam ser feitas pelos cirurgiões ${ }^{8}$.

Existem dados mais concretos sobre o retorno ao esporte após a prótese unicompartimental de joelho ${ }^{23,24}$. Inclusive ao comparar-se o procedimento de ATJ com essa. Mas não foi o mote desse estudo a prótese unicondilar, o que abre um caminho para futuras pesquisas e observações.

Diante da ausência de estudos de maior nível de evidência como os ensaios clínicos randomizados, uma das limitações desta revisão foi a inclusão apenas de estudos retrospectivos, e com potencial risco de viés devido à forma de avaliação dos desfechos por telefone ou via e-mail; todos os estudos incluídos relataram perdas de pacientes no seguimento maiores de $45 \%$.

\section{CONSIDERAÇÕES FINAIS}

Os estudos incluídos nessa revisão mostraram que a maioria dos pacientes que participou de alguma atividade física regular antes da artroplastia total de joelho (ATJ) retornou à prática esportiva após a cirurgia, sendo mais comuns os esportes recreacionais de baixo impacto como caminhada, esportes aquáticos, golfe, bicicleta estacionária, entre outros. Foi possível observar que o nível de atividade física não depende da dor e dos sintomas pós-operatórios, mas da satisfação com a cirurgia, motivação e capacidade funcional dos indivíduos. Não foi possível analisar os efeitos reais da atividade esportiva sobre o desgaste da prótese devido à falta de relato dos estudos. O tempo de retorno ao esporte foi descrito por apenas três estudos e variou de seis a 18 meses após a ATJ. Apesar de alguns estudos mostrarem que não há recomendação por parte dos cirurgiões para a prática de esportes de alto impacto após a cirurgia, alguns estudos mostraram bons resultados, desde que se considere o nível de atividade prévio à cirurgia, a população adequada e a expectativa do paciente no pós-operatório. Assim, conclui-se que o retorno ao esporte após ATJ não só é possível, como é recomendado em níveis recreativos, e um paciente colaborativo, instruído e com preparo adequado de condicionamento físico pode melhorar tanto a nível funcional e cardiovascular quanto psicológico, com melhora da autoestima e da qualidade de vida. Há ainda a necessidade de estudos futuros, de boa qualidade metodológica como os ensaios clínicos randomizados, sobre os efeitos (benefícios e prejuízos) dos esportes de alto impacto para os pacientes com ATJ. Há ainda a necessidade de estudos futuros, de boa qualidade metodológica como os ensaios clínicos randomizados, sobre os efeitos (benefícios e prejuízos) dos esportes de alto impacto para os pacientes com ATJ.

Todos os autores declararam não haver qualquer potencial conflito de interesses referente a este artigo.

CONTRIBUIÇÕES DOS AUTORES: Cada autor contribuiu individual e significativamente para o desenvolvimento do manuscrito. PBJ (0000-0002-4444-4004)* e APSF 0000-0002-8927-095x)* foram os principais contribuintes na redação do manuscrito. PBJ e RYM (0000-0002-2890-9860)* foram responsáveis pela revisão da literatura. GAEP (0000-0002-1316-3840)*, JWCS (0000-0002-9239-1736)*, LGBG (0000-0002-6946-1628)* e MVL (0000-0002-6946-1628)* auxiliaram na manufatura e revisão do manuscrito. ADJ (0000-0001-8201-0701)* e RPLC (0000-0003-0507-4976)* contribuíram com o conceito intelectual do estudo. ${ }^{*}$ ORCID (Open Researcher and Contributor ID). 


\section{REFERÊNCIAS}

1. Healy WL, Sharma S, Schwartz B, lorio R. Athletic activity after total joint arthroplasty. J Bone Joint Surg Am. 2008;90(10):2245-52.

2. Schmalzried TP, Shepherd EF, Dorey FJ, Jackson WO, dela Rosa M, Fa'vae F, et al. The John Charnley Award. Wear is a function of use, not time. Clin Orthop Relat Res. 2000;(381):36-46.

3. D'Lima DD, Patil S, Steklov N, Slamin JE, Colwell CW Jr. Tibial forces measured in vivo after total knee arthroplasty. J Arthroplasty. 2006;21(2):255-62.

4. Lavernia CJ, Sierra RJ, Hungerford DS, Krackow K. Activity level and wear in total knee arthroplasty: a study of autopsy retrieved specimens. J Arthroplasty. 2001;16(4):446-53.

5. Jassim SS, Douglas SL, Haddad FS. Athletic activity after lower limb arthroplasty: a systematic review of current evidence. Bone Joint J. 2014;96-B(7):923-7.

6. Howick J, Chalmers I, Glasziou P, Greenhalgh T, Heneghan C, Liberati A, et al. "Explanation of the 2011 Oxford Centre for Evidence-Based Medicine (OCEBM) Levels of Evidence (Background Document)". Oxford Centre for Evidence-Based Medicine. Disponível em: http://www.cebm.net/wp-content/ uploads/2014/06/CEBM-Levels-of-Evidence-Background-Document-2.1.pdf

7. Chang MJ, Kim SH, Kang YG, Chang CB, Kim TK. Activity levels and participation in physical activities by Korean patients following total knee arthroplasty. BMC Musculoskelet Disord. 2014;15:240.

8. Bonnin M, Laurent JR, Parratte S, Zadegan F, Badet R, Bissery A. Can patients really do sport after TKA? Knee Surg Sports Traumatol Arthrosc. 2010;18(7):853-62.

9. Jackson JD, Smith J, Shah JP, Wisniewski SJ, Dahm DL. Golf after total knee arthroplasty: do patients return to walking the course? Am J Sports Med. 2009;37(11):2201-4.

10. Mont MA, Marker DR, Seyler TM, Jones LC, Kolisek FR, Hungerford DS. High-impact sports after total knee arthroplasty. J Arthroplasty. 2008;23(6 Suppl 1):80-4.

11. Dahm DL, Barnes SA, Harrington JR, Sayeed SA, Berry DJ. Patient-reported activity level after total knee arthroplasty. J Arthroplasty. 2008;23(3):401-7.

12. Chatterji U, Ashworth MJ, Lewis PL, Dobson PJ. Effect of total knee arthroplasty on recreational and sporting activity. ANZ J Surg. 2005;75(6):405-8.
13. Mont MA, Rajadhyaksha AD, Marxen JL, Silberstein CE, Hungerford DS. Tennis after total knee arthroplasty. Am J Sports Med. 2002;30(2):163-6.

14. Bradbury N, Borton D, Spoo G, Cross MJ. Participation in sports after total knee replacement. Am J Sports Med. 1998;26(4):530-5.

15. Diduch DR, Insall JN, Scott WN, Scuderi GR, Font-Rodriguez D. Total knee replacement in young, active patients. Long-term follow-up and functional outcome. J Bone Joint Surg Am. 1997;79(4):575-82.

16. Ries MD, Philbin EF, Groff GD, Sheesley KA, Richman JA, Lynch F Jr. Improvement in cardiovascular fitness after total knee arthroplasty. J Bone Joint Surg Am. 1996;78(11):1696-701.

17. Mallon WJ, Callaghan JJ. Total knee arthroplasty in active golfers. J Arthroplasty. 1993;8(3):299-306.

18. Seyler TM, Mont MA, Ragland PS, Kachwala MM, Delanois RE. Sports activity after total hip and knee arthroplasty : specific recommendations concerning tennis. Sports Med. 2006;36(7):571-83.

19. Chillag KJ, Barth E. An analysis of polyethylene thickness in modular total knee components. Clin Orthop Relat Res. 1991;(273):261-3.

20. Feng EL, Stulberg SD, Wixson RL. Progressive subluxation and polyethylene wear in total knee replacements with flat articular surfaces. Clin Orthop Relat Res. 1994;(299):60-71.

21. lorio R, Healy WL, Applegate T.Validity of preoperative demand matching as an indicator of activity after TKA. Clin Orthop Relat Res. 2006;452:44-8.

22. Barber-Westin SD, Noyes FR. Aerobic Physical Fitness and Recreational Sports Participation After Total Knee Arthroplasty: A Systematic Review. Sports Health. 2016 Sep 19. pii: 1941738116670090 . [Epub ahead of print] PubMed PMID: 27644234.

23. Ho JC, Stitzlein RN, Green CJ, Stoner T, Froimson MI. Return to Sports Activity following UKA and TKA. J Knee Surg. 2016;29(3):254-9.

24. Witjes S, Gouttebarge V, Kuijer PP, van Geenen RC, Poolman RW, Kerkhoffs GM. Return to Sports and Physical Activity After Total and Unicondylar Knee Arthroplasty: A Systematic Review and Meta-Analysis. Sports Med. 2016;46(2):269-92. 